\section{Validation of printed and computerised crowded Kay picture logMAR tests against gold standard ETDRS acuity test chart measurements in adult and amblyopic paediatric subjects}

N Shah ${ }^{1}$, DAH Laidlaw ${ }^{1}$, S Rashid ${ }^{1}$ and P Hysi ${ }^{2}$

when compared with the gold standard ETDRS. Measurement error means that differences of up to $0.16 \log$ MAR may be observed in clinically stable patients when re-measured using the same technique. A combination of TRV and systematic bias can however lead to differences of up to $\mathbf{0 . 4 0}$ $\log$ MAR in stable amblyopic patients when switched from CKPs to ETDRS chart acuity measurements.

Eye (2012) 26, 593-600; doi:10.1038/eye.2011.333; published online 23 December 2011

Keywords: visual acuity; Kay picture test; COMPlog; logMAR

\section{Introduction}

Kay picture tests are used for measurement of visual acuity in illiterate children and adults, and are available in crowded $\log$ MAR and Snellen formats. ${ }^{1}$ The tests have however been subject to only limited validation against gold standard letter acuity tests. Furthermore, this validation involved correlation of the Kay picture and letter acuity test results. ${ }^{1-3}$ The finding of a significant correlation in these circumstances informs us that one measurement increases linearly with the other, but reveals nothing about the degree of agreement (or disagreement) between test results. It also does not reveal any systematic or proportional bias
${ }^{1}$ Department of Ophthalmology, St Thomas' Hospital, London, UK

${ }^{2}$ Department of Twin Research \& Genetic Epidemiology, King's College, London, UK

Correspondence: DAH Laidlaw, Department of Ophthalmology, St Thomas' Hospital, Lambeth Palace Road, London SE1 7EH, UK Tel: +44 (0)20 7188 4320; Fax: +44 (0)20 71889193. E-mail: Alistair.Laidlaw@ gstt.nhs.uk

Received: 20 May 2011 Accepted in revised form: 14 November 2011 Published online: 23 December 2011 
between the underlying acuity and extent of agreement. There is as yet an unsubstantiated clinical impression amongst users of Kay pictures that they over read acuity (that is, produce better test results) than is measured on the same patient with letter acuity charts (RA Harrad, personal communication, 2008). Many children will transfer from picture to letter acuity tests as they mature, and it is important that any test professing a Snellen or $\log$ MAR score should be comparable against the recognised standard metric.

An important index of the performance of a clinical test is assessment of the error inherent in that measurement. For acuity tests, this is usually expressed as a Bland-Altman $95 \%$ limit of agreement. ${ }^{4}$ These limits of agreement quantify the magnitude of normal test re-test variability (TRV) in measurements, which are taken on the same subject in the same way when no clinical change has occurred. For ETDRS logMAR acuity charts, changes of up to $10 \log$ MAR letters ( 2 logMAR lines) in either direction are recognised to occur. ${ }^{5-15}$ This aspect of the performance of Kay picture acuity measurements has also not previously been investigated.

The development of computerised acuity measurement devices such as the E-ETDRS algorithm ${ }^{8,16,17}$ and COMPlog visual acuity measurement system, ${ }^{5,6}$ as well as computerised test chart presentation devices such as Test Chart 2000, means the reliability of computerised presentation of test stimuli should be investigated.

The aims of this validation study in two different study groups were:

(1) To investigate for bias between pCKP and cCKP $\log$ MAR acuity measurements when compared with each other and with the gold standard single letter scoring ETDRS chart acuity measurements.

(2) To compare the TRV of hard copy and computerised $\log$ MAR Kay picture acuity measurements and ETDRS chart acuity measurements.

\section{Materials and methods}

A sample of 30 adult subjects with various ophthalmic diseases were recruited from the Adult Outpatient Clinics and 40 patients were recruited from the Paediatric Orthoptic clinic, with either strabismic, anisometropic, or mixed amblyopia. The exclusion criteria for this study were the inability to read letters and a visual acuity of $1.00 \log$ MAR (6/60) or worse measured with letter optotypes using the COMPlog visual acuity measurement system.

Each subject underwent single-letter scoring test and retest measurements of the visual acuity of their weaker or amblyopic eye, using ETDRS charts 1 and 2, printed
Kay picture tests (pCKP) versions 1 and 2, and the Kay pictures testing algorithm on the COMPlog system (cCKP), that is, six tests in total. These measurements are referred to as ETDRS1, ETDRS2, pCKP1, pCKP2, cCKP1, and CCKP2. All tests were performed in a random sequence to control for fatigue and learning effects, with the random sequence being generated by Research Randomizer (http://www.randomizer.org/). All measurements were conducted by one trained examiner (NS) under consistent lighting conditions. Subjects wore their habitual spectacle correction with their fellow eye occluded. Responses and computerised test scores were recorded on specifically designed proformata.

ETDRS charts 1 and 2 were used and displayed in the standard Lighthouse Low Vision Products light box (Lighthouse Low Vision Products, Long Island City, NY, USA). The ETDRS charts were read from a distance of $4 \mathrm{~m}$.

Printed Crowded logMAR Kay Picture Tests 1 and 2 (KAY PICTURES, Tring, UK) were used and were viewed from their design distance of $3 \mathrm{~m}$.

COMPlog is a computerised visual acuity measurement system consisting of a laptop PC capable of running Microsoft Windows XP or subsequent operating systems, a 21.3 inch $1600 \times 1200$ resolution LCD flat panel secondary monitor, and the COMPlog software programme running within the Microsoft dotnet framework. It is capable of displaying both letter and picture optotypes. The COMPlog Testing algorithm consists of two phases: 'range finding' and 'thresholding', which has been described in detail in previous publications. ${ }^{5,6}$ Essentially, the range-finding phase presents single optotypes surrounded by a crowding box to determine approximate threshold acuity. The thresholding phase then displays sequentially smaller single lines of optotypes surrounded by a crowding box. In the event of optotypes being incorrectly identified on the first thresholding line, sequentially larger lines are presented until an entire line is correctly read, with the programme then descending to threshold, but only presenting lines of each size once. If a subject is unable to identify a $1.60 \log$ MAR size optotype, the programme invites scoring on a count finger, hand movements, perception of light, and no perception of light scale. In this way, with a 21.3-inch monitor and a single viewing distance of $3 \mathrm{~m}$, a letter acuity range of between -0.30 and $1.68 \log$ MAR (100 to 1 ETDRS letters, $6 / 3$ to $1.5 / 71$ UK Snellen) may be measured without moving the patient.

In this study, each test line consisted of four randomly selected Kay pictures with no repeats on any line, spaced half a picture width apart and surrounded at the same separation by a crowding box of one stroke width thickness. The termination criterion was set at all four 
pictures on one line. If all four Kay picture optotypes could not fit simultaneously on to the secondary monitor, the line was broken up into fractions with as many pictures as possible of each size being presented, always surrounded by a crowding box. Once the termination criterion had been met, the test automatically terminated with calculation and presentation of a fully interpolated $\operatorname{logMAR}$ acuity score and its Snellen equivalent in the selected formats.

Kay picture images were scanned using commercial scanning hard and software at $500 \mathrm{dpi}$. This resulted in a digital resolution, which was greater than the screen pixel size of $0.27 \mathrm{dpi}$ at the set viewing distance of $3 \mathrm{~m}$.

The end point for all tests employed in this study was defined as an entire line of letters/pictures being misread. A fully interpolated letter-by-letter logMAR acuity score was calculated for each test result in which credit was given for each test optotype correctly identified. ${ }^{14,18,19}$

The COMPlog secondary monitor had a screen luminance of $288 \mathrm{~cd} / \mathrm{m}^{2}$ and the contrast of letters was measured to be $99.8 \%$. The ETDRS-employed chart was measured to have a luminance of $111 \mathrm{~cd} / \mathrm{m}^{2}$.

Ethical approval for this study was granted from St Thomas' Hospital Research Ethics Committee, and informed consent was obtained from each subject or if appropriate, their parents or carers. We certify that all applicable institutional and governmental regulations concerning the ethical use of human volunteers were followed during this research.

\section{Statistical analysis}

Descriptive statistics, the methods of Band and Altman, ${ }^{4}$ and Deming regression ${ }^{20}$ were employed. The GraphPad
Prism statistical analysis package (GraphPad Software Inc., La Jolla, CA, USA) was employed for this purpose.

\section{Results}

A total of 30 adult subjects aged between 30 and 83 (mean 59.8 years) were recruited and consented to participate in the study: 17 with retinal problems, 3 with glaucoma/optic neuropathy, and 10 with mixed pathology. A second group of 40 children aged between 4 years and 15 years (mean 8 years) with amblyopia was also recruited. Of these 40 children, 25 had strabismic amblyopia, 13 had anisometropic amblyopia, and 2 had a combination of two aetiologies of amblyopia.

The first test mean ETDRS letter acuity was 0.44 logMAR, range 0.10 logMAR-0.96 zogMAR, SD 0.25 (UK Snellen Equivalents 6/16.5, 6/7.6 to 6/54.7) for the adult subset and $0.48 \log$ MAR, range 0.14-1.00 logMAR, SD 0.22 (UK Snellen Equivalents 6/18.1, 6/8.3 to 6/60) for the paediatric group. Data sets were examined and found to conform reasonably to a normal distribution.

Bland and Altman scatter plots were constructed to represent the graphical spread of each of the six pairs of measurement results in each of the two study groups: these were ETDRS 1 vs ETDRS 2, pCKP1 vs pCKP2, cCKP1 vs cCKP2, pCKP1 vs cCKP1, ETDRS1 vs pCKP1, and ETDRS1 vs cCKP1. The first three series in each group demonstrate the TRV of each measurement technique. The latter three are method comparison studies. In each case, the mean difference and upper and lower $95 \%$ limits of agreement are plotted as dotted lines.

In each of the six test-retest series, there was no evidence of any systematic association between the level of agreement and the underlying acuity (ie, Figures 1-3, the ETDRS 1 vs ETDRS 2, pCKP1 vs PCKP2, and cCKP1 vs cCKP2 comparisons in the adult and paediatric groups). a

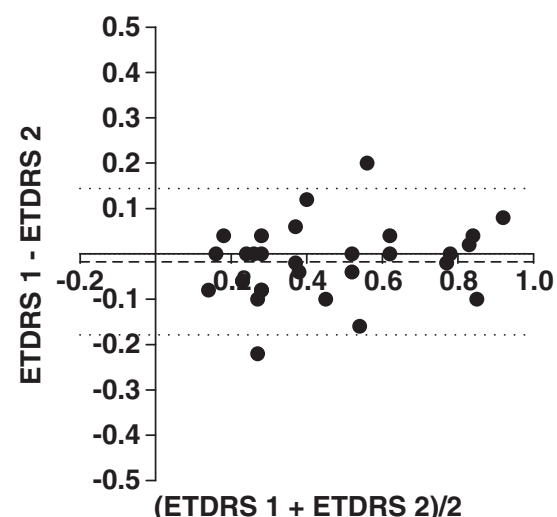

b

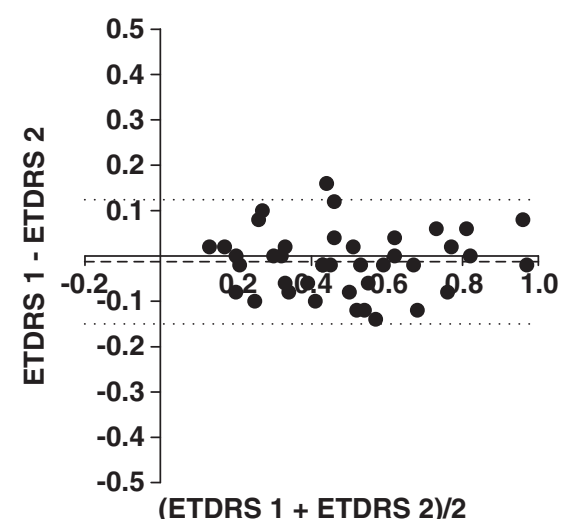

Figure 1 Bland-Altman plot for test and retest measurements in the (a) adult and (b) paediatric groups of ETDRS chart acuity measurements. In each instance, the mean difference and upper and lower 95\% limits of agreement are plotted. 
a

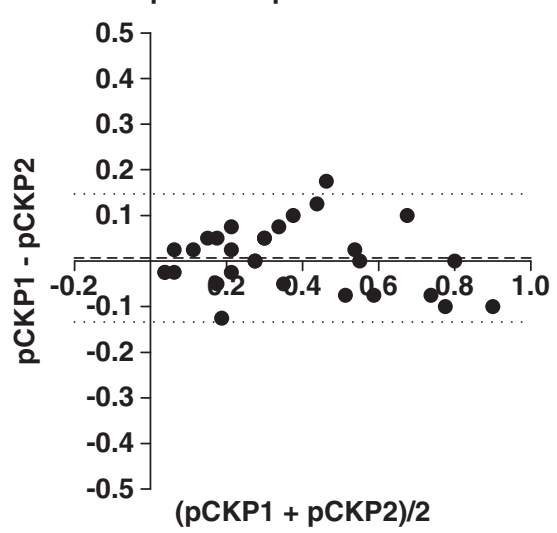

b pCKP1 v pCKP2 Children

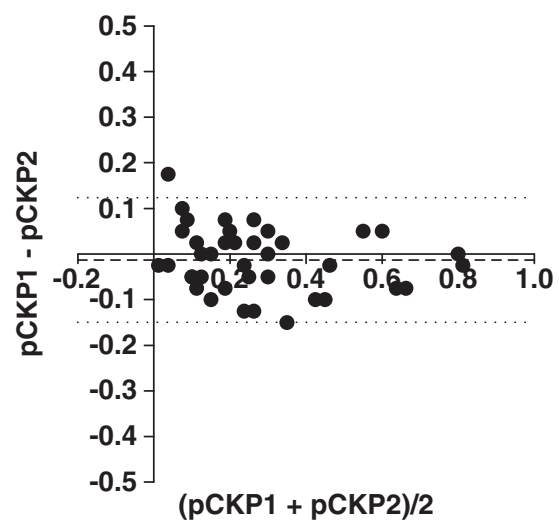

Figure 2 Bland-Altman plot for test and retest measurements in the (a) adult and (b) paediatric groups of pCKP acuity measurements. In each instance, the mean difference and upper and lower 95\% limits of agreement are plotted.

a

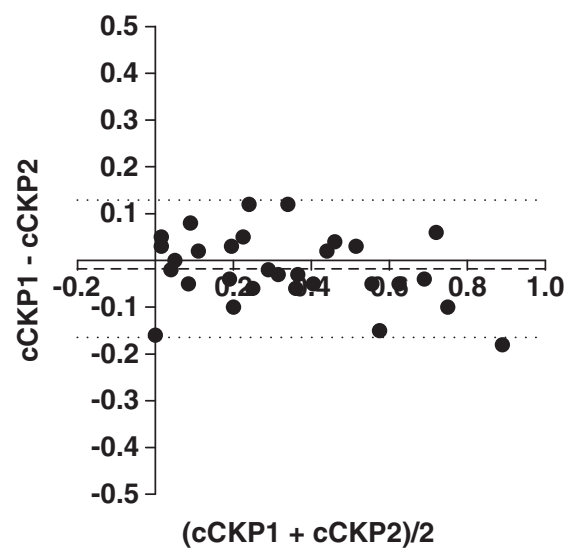

b $\quad$ cCKP1 v cCKP2 Children

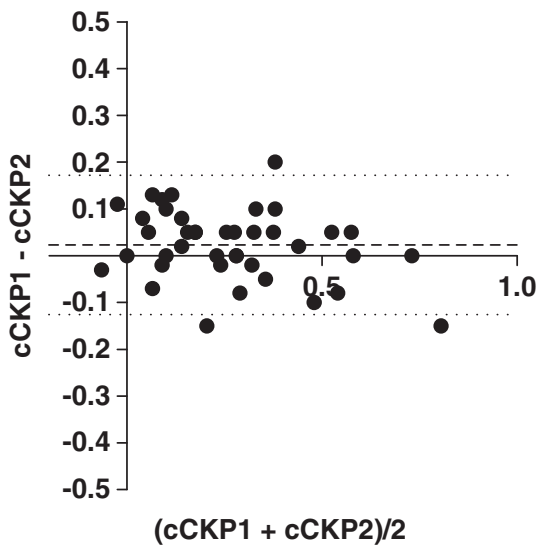

Figure 3 Bland-Altman plot for test and retest measurements in the (a) adult and (b) paediatric groups of cCKP acuity measurements. In each instance, the mean difference and upper and lower 95\% limits of agreement are plotted.

Table 1 The mean bias and where relevant, TRV expressed as 95\% limits of agreement for each of the six test pairs in the adult group in $\log$ MAR

\begin{tabular}{lcccc}
\hline & $\begin{array}{c}\text { Mean difference } \\
(S E)\end{array}$ & $\begin{array}{c}\text { 95\% CI mean } \\
\text { difference }\end{array}$ & $\begin{array}{c}\text { Range of observed } \\
\text { differences }\end{array}$ & $\begin{array}{c}\text { TRV (95\% limits } \\
\text { of agreement) }\end{array}$ \\
\hline ETDRS 1-ETDRS 2 & $-0.02(0.02)$ & $-0.05,0.01$ & $-0.22,0.20$ & \pm 0.16 \\
pCKP 1-pCKP 2 & $0.01(0.01)$ & $-0.02,0.04$ & $-0.13,0.18$ & \pm 0.14 \\
cCKP 1-cCKP 2 & $-0.02(0.01)$ & $-0.05,0.01$ & $-0.18,0.12$ & \pm 0.14 \\
ETDRS 1-pCKP 1 & $0.08(0.02)$ & $0.04,0.12$ & $-0.19,0.24$ & \pm 0.19 \\
ETDRS 1-cCKP 1 & $0.12(0.02)$ & $0.08,0.16$ & $-0.14,0.38$ & \pm 0.21 \\
pCKP 1-cCKP 1 & $0.04(0.01)$ & $0.01,0.07$ & $-0.11,0.22$ & \pm 0.14 \\
\hline
\end{tabular}

These data are presented graphically in Figures 1a, 2a, 3a, 4a, 5a, and 6a.

The TRV data were also found in each case to conform reasonably to a normal distribution. On this basis, Bland and Altman summary statistics of mean bias and 95\% limits of agreement were calculated and are presented in Table 1 and Table 2 for the adult group and the paediatric group, respectively.
Similar results suggesting no clinically significant bias and comparable TRV were found for the method comparison study between the computerised and printed formats of CKP acuity measurements (ie, Figure 4, pCKP1 vs cCKP1) in both groups. These data are presented in row 7 of Tables 1 and 2 . 
Table 2 The mean bias and where relevant, TRV expressed as 95\% limits of agreement for each of the six relevant test pairs in the paediatric group in logMAR

\begin{tabular}{lcccc}
\hline & $\begin{array}{c}\text { Mean difference } \\
(\text { SE })\end{array}$ & $\begin{array}{c}\text { 95\% CI mean } \\
\text { difference }\end{array}$ & $\begin{array}{c}\text { Range of observed } \\
\text { differences }\end{array}$ & $\begin{array}{c}\text { TRV (95\% limits } \\
\text { for agreement) }\end{array}$ \\
\hline ETDRS 1-ETDRS 2 & $-0.01(0.01)$ & $-0.03,0.01$ & $-0.14,0.16$ & \pm 0.14 \\
PCKP 1-pCKP 2 & $-0.01(0.01)$ & $-0.03,0.01$ & $-0.15,0.18$ & \pm 0.14 \\
CCKP 1-cCKP 2 & $0.02(0.01)$ & $0.00,0.04$ & $-0.15,0.20$ & \pm 0.16 \\
ETDRS 1-pCKP 1 & $0.22(0.01)$ & $0.19,0.25$ & $0.04,0.42$ & \pm 0.16 \\
ETDRS 1-cCKP 1 & $0.20(0.01)$ & $0.17,0.23$ & $-0.08,0.36$ & \pm 0.18 \\
pCKP 1-cCKP 1 & $-0.01(0.01)$ & $-0.04,0.02$ & $-0.28,0.21$ & \pm 0.18 \\
\hline
\end{tabular}

These data are presented graphically in Figures $1 b, 2 b, 3 b, 4 b, 5 b$, and $6 b$.
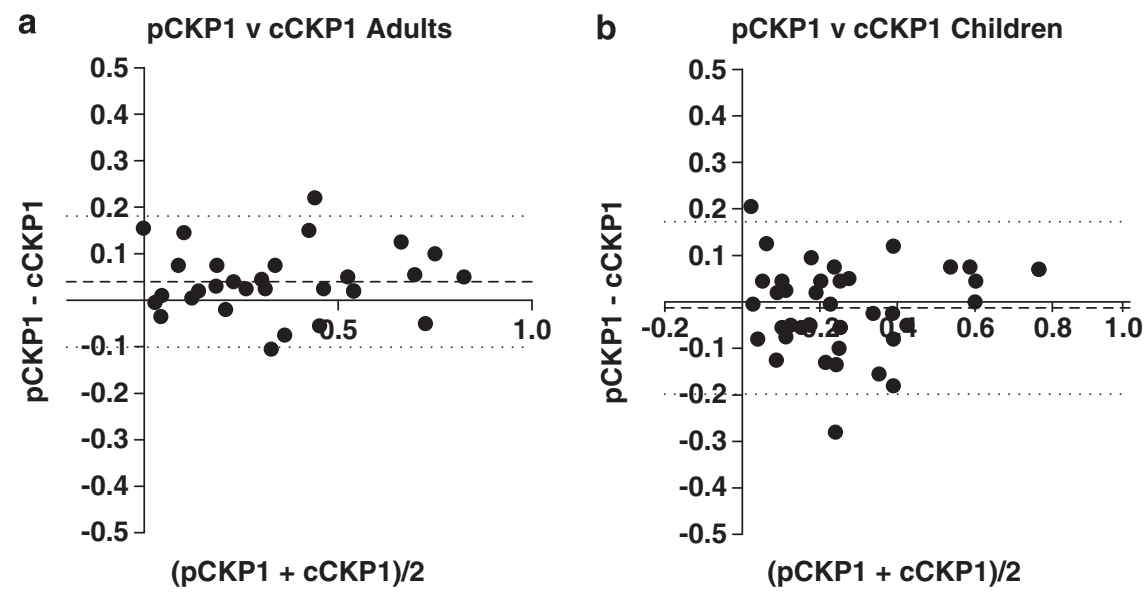

Figure 4 Bland-Altman plots for method comparison studies between pCKP and cCKP acuity measurements in the (a) adult group and (b) paediatric groups. In each instance, the mean difference and upper and lower $95 \%$ limits of agreement are plotted.

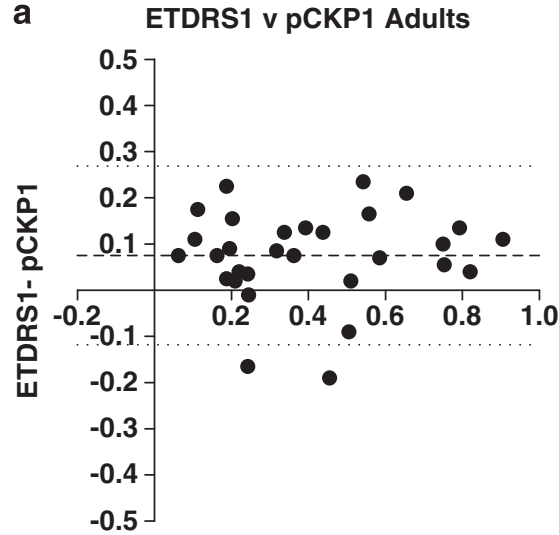

(ETDRS1+pCKP1)/2

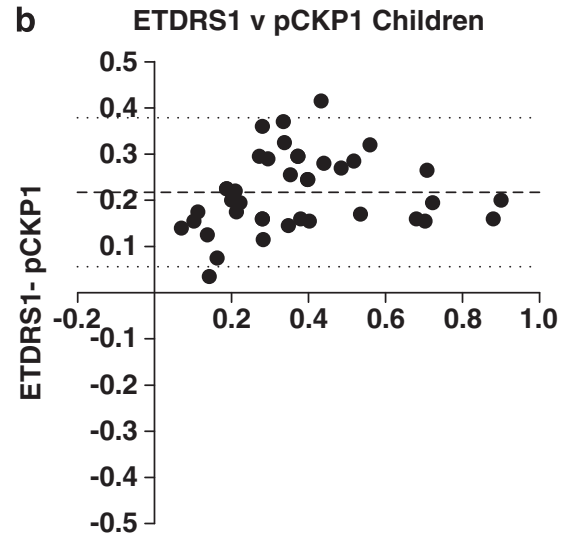

(ETDRS1+pCKP1)/2

Figure 5 Bland-Altman plots for method comparison studies between ETDRS and pCKP acuity measurements in the (a) adult group and (b) paediatric groups. In each instance, the mean difference and upper and lower 95\% limits of agreement are plotted.

The Bland-Altman scatter plots comparing ETDRS chart and both cCKP and pCKP acuity measurements are shown in Figures 5 and 6. In each series, there is evidence of bias with the Kay picture values consistently producing better acuity scores. A proportional as well as systematic bias might also have been inferred from interpretation of Figures $5 b$ and $6 b$, which is a greater level of disagreement between measurements in patients 
a

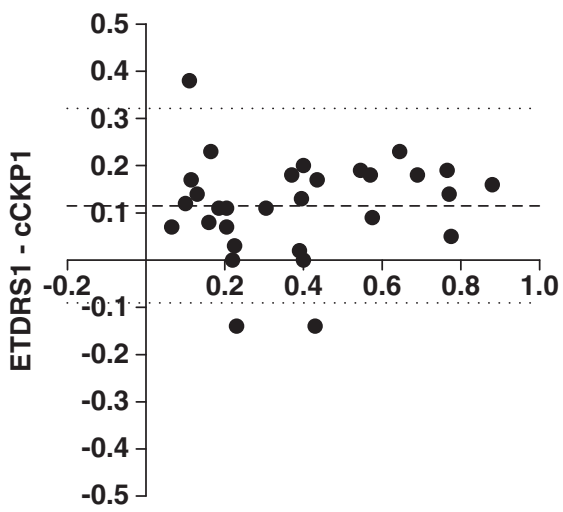

(ETDRS1+cCKP1)/2

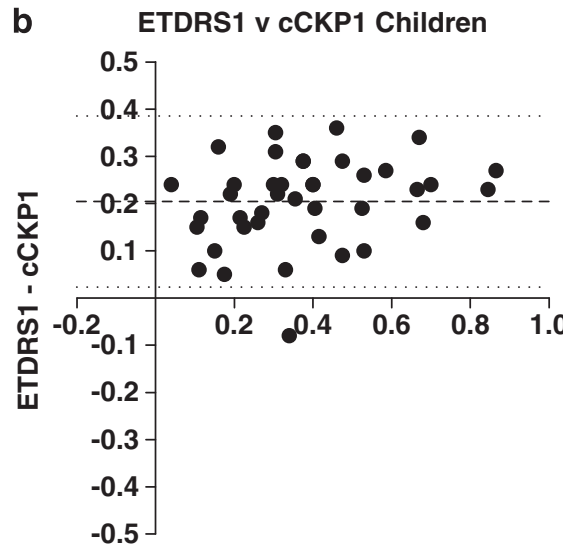

(ETDRS1+cCKP1)/2

Figure 6 Bland-Altman plots for method comparison studies between ETDRS and cCKP acuity measurements in the (a) adult group and (b) paediatric groups. In each instance, the mean difference and upper and lower $95 \%$ limits of agreement are plotted.

Table 3 Results of Deming regression analysis to investigate for proportional, as well as systematic bias between ETDRS chart and crowded Kay Picture acuity measurements

\begin{tabular}{lccc}
\hline Model & $\begin{array}{c}\text { Deming regression slope } \\
\text { (SE) }\end{array}$ & $\begin{array}{c}\text { 95\% CI slope } \\
\text { 95\% CI y intercept when } \\
\mathrm{x}=0\end{array}$ \\
\hline Mean ETDRS acuity $(x)$, mean pCKP $(y)$ (adults) & $1.04(0.06)$ & $0.91,1.17$ & $-0.17,-0.04$ \\
Mean ETDRS acuity $(x)$, mean CCKP $(y)$ (adults) & $1.05(0.08)$ & $0.89,1.21$ & $-0.22,-0.06$ \\
Mean ETDRS acuity $(x)$, mean pCKP $(y)$ (paediatric amblyopes) & $0.97(0.06)$ & $0.85,1.09$ & $-0.27,-0.14$ \\
Mean ETDRS acuity $(x)$, mean CCKP $(y)$ (paediatric amblyopes) & $0.95(0.06)$ & $0.83,1.06$ & $-0.26,-0.13$ \\
\hline
\end{tabular}

In each case, the mean of the test and retest measurements with each technique has been employed.

with worse acuity. The results of Deming regression performed to investigate for such a proportional bias are presented in Table 3. In each case, to minimise the effect of measurement error when performing this regression, the mean of the test and retest measurements taken with each technique was employed.

In the absence of consistent clinically significant evidence of a proportional bias, Bland-Altman limits of agreement for the method comparison between ETDRS chart measurements and cCKP and pCKP acuity measurements are presented in Tables 1 and 2 .

\section{Discussion}

Computerised and printed Kay picture acuity measurements show consistently similar TRV to that observed on the same subjects using an ETDRS chart when measured in both adults and amblyopic children (either \pm 0.14 or $\pm 0.16 \log$ MAR with each test, Tables 1 and 2, rows 2-4). These results for each technique are typical of those published in other studies of the TRV of $\log$ MAR acuity measurements ${ }^{5-15}$ in which TRV of acuity measurements of up to $0.2 \log$ MAR (two logMAR lines) are observed in patients with unchanged acuity. These data suggest that the CKP tests are similarly repeatable to ETDRS chart acuity measurements.

No systematic bias was evident between printed and computerised $\log$ MAR Kay picture acuity measurements in the paediatric amblyopic group (mean difference $-0.01,95 \%$ confidence interval mean difference -0.04 , $0.02 \log$ MAR (Table 2, row 7). In the adult population, the 95\% confidence interval of the mean difference of 0.04 $\log$ MAR only just misses $0(0.01,0.07)$. Whether this is a significant systematic bias or a chance finding in a study with multiple different outcome measures will be explored in future studies. It should be appreciated however that this potential two-letter bias is occurring in the presence of normal TRV of up to two lines in either direction. ${ }^{5-15}$ On this basis, it does not seem unreasonable to conclude that measurements taken with the computerised and printed versions of the CKP acuity test might be considered clinically interchangeable.

The difficulty arises when trying to relate a CKP acuity score to its equivalent measured with the gold standard ETDRS letter chart, as might be required when children mature and are transferred to letter acuity. The BlandAltman scatter plots of the comparison between ETDRS acuity measurements and both pCKP and cCKP acuity 
scores are presented in Figures 5 and 6. A clear systematic bias is present in each series with the Kay picture acuity scores consistently producing better acuity than the ETDRS chart measurements. The scatter of the points in the paediatric group (Figures $5 \mathrm{~b}$ and $6 \mathrm{~b}$ ) might also suggest that the level of mean disagreement between measurements in these amblyopic subjects increased with worsening acuity (ie, a line of best fit might have a positive slope). The methods described by Bland and Altman do not address the investigation of such a potential proportional bias. ${ }^{4}$ We therefore performed Deming regression ${ }^{20}$ of the mean ETDRS acuity score against the mean CKP acuity scores to investigate this issue. This is a maximum likelihood test and addresses the potential uncertainty in the variables under scrutiny, and has been advocated for use in ophthalmic method comparison studies. ${ }^{21}$ The results are presented in Table 3. No significant proportional bias was found in each series, that is, the level of mean disagreement between measurements in both the adult and paediatric group were not found to increase with worsening acuity. We therefore calculated Bland and Altman mean and 95\% limits of agreement for testing the method comparison between ETDRS chart and pCKP and cCKP acuity scores. It can be seen that in adults, the Kay picture acuity measurements were consistently better by approximately one logMAR line (0.08 and $0.12 \log$ MAR, respectively, for the printed and computerised formats) and by approximately two logMAR lines in the amblyopic children (0.22 and $0.20 \log$ MAR, respectively).

These data suggesting that picture acuity

measurements overestimate acuity when compared with letter charts in children are not unique. Dobson et $a^{22}$ found mean Lea Symbols visual acuity was half a logMAR line better than ETDRS visual acuity in a population of young children with a high prevalence of astigmatism. They also found Lea Symbols acuity scores were one logMAR line better than Bailey-Lovie acuity scores, and this difference increased with worse visual acuity in school-aged children and adults. ${ }^{23}$

There may be a number of contributing factors to the difference in acuities measured with picture vs letter optotypes. The Kay picture test is effectively an 8 alternative-forced choice test compared with the ETDRS chart, which is a 10 alternative-forced choice test. Carkeet $^{24}$ found that the mean and SD of logMAR scores was significantly affected by the number of forced choice alternatives as have other studies. ${ }^{24,25}$ The interest of young children was possibly also better maintained with the more familiar picture optotypes than letters, which may explain the greater mean difference in acuity between the two test types in children compared with adults. An alternative explanation is that the CKP test is systematically more crowded than the ETDRS chart, hence the observed greater bias in amblyopic children than adults without known amblyopia.

These results are observational in nature and need confirmation in future studies. However, three provisional conclusions may be drawn:

(1) If both systematic bias and TRV are taken into account, a logMAR CKP acuity measurement in an amblyopic child may be up to four logMAR lines better than an ETDRS chart score in the same subject. If this effect in presented in Snellen equivalent terms, it would be equivalent to measurements of $6 / 6$ and $6 / 15$, or $6 / 24$ and 6/60, respectively, with a CKP and ETDRS chart. On average scores, Kay picture acuity scores would be two logMAR lines better than ETDRS chart scores. The variation due to measurement error if the ETDRS or CKP tests are repeated is up to one and a half lines.

(2) In the absence of any change in acuity, a logMAR CKP score in an amblyopic child is unlikely to be worse than an ETDRS score.

(3) It may be reasonable to mathematically convert $\log$ MAR CKP acuity scores to an ETDRS letter chart equivalent; however, further studies are required to confirm these observations.

\section{Summary}

\section{What was known before}

- Kay picture tests have been subject to only limited validation against gold standard letter acuity tests.

What this study adds

- This study confirms through appropriate and controlled testing what many clinicians have thought, but has not previously been verified, that visual acuity scores attained with the Kay picture test are biased compared with the ETDRS chart, giving better scores.

\section{Conflict of interest}

DAH Laidlaw and Guys and St Thomas' Foundation NHS Trust have proprietary interests in the COMPlog visual acuity measurement system. All other authors declare no conflict of interest.

\section{References}

1 Kay H. New method of assessing visual acuity with pictures. Br J Ophthalmol 1983; 67: 131-133.

2 Jones D, Westall C, Averbeck K, Abdolell M. Visual acuity assessment: a comparison of two tests for measuring children's vision. Ophthal Physiol Opt 2003; 23: 541-546.

3 Elliott MC, Firth AY. The logMAR Kay picture test and the logMAR acuity test: a comparative study. Eye 2009; 23: 85-88. 
4 Bland JM, Altman DG. Statistical methods for assessing agreement between two methods of clinical measurement. Lancet 1986; 1: 307-310.

5 Laidlaw DAH, Tailor V, Shah N, Atamian S, Harcourt C. Validation of a computerised logMAR visual acuity measurement system (COMPlog): comparison with ETDRS and the Electronic ETDRS testing algorithm in adults and amblyopic children. Br J Ophthalmol 2008; 92: 241-244.

6 Shah N, Laidlaw DAH, Brown G, Robson C. Effect of letter separation on computerised visual acuity measurements: comparison with the gold standard Early Treatment Diabetic Retinopathy Study (ETDRS) chart. Ophthal Physiol Opt 2010; 30: 200-203.

7 Laidlaw DAH, Abbott A, Rosser DA. Development of a clinically feasible $\log$ MAR alternative to the Snellen chart: performance of the 'compact reduced logMAR' visual acuity chart in amblyopic children. Br J Ophthalmol 2003; 87: 1232-1234.

8 Beck RW, Moke PS, Turpin AH, Ferris 3rd Fl, SanGiovanni JP, Johnson CA. A computerized method of visual acuity testing: adaptation of the early treatment of diabetic retinopathy study testing protocol. Am J Ophthalmol 2003; 135: 194-205.

9 Rosser DA, Laidlaw DAH, Murdoch IE. The development of a 'reduced $\log M A R^{\prime}$ visual acuity chart for use in routine clinical practice. Br J Ophthalmol 2001; 85: 432-436.

10 Siderov J, Tiu AL. Variability of measurements of visual acuity in a large eye clinic. Acta Ophthalmol Scand 1999; 77: 673-676.

11 Lovie-Kitchin JE. Validity and reliability of visual acuity measurements. Ophthalmic Physiol Opt 1988; 8: 363-370.

12 Reeves BC, Wood JM, Hill AR. Vistech VCTS 6500 charts within- and between-session reliability. Optom Vis Sci 1991 68: 728-737.

13 Elliott DB, Sheridan M. The use of accurate visual acuity measurements in clinical anti-cataract formulation trials. Ophthalmic Physiol Opt 1988; 8: 397-401.
14 Arditi A, Cagenello R. On the statistical reliability of letterchart visual acuity measurements. Invest Ophthalmol Vis Sci 1993; 34: 120-129.

15 Reeves BC, Wood JM, Hill AR. Reliability of high- and lowcontrast letter charts. Ophthalmic Physiol Opt 1993; 13: 17-26.

16 Cotter SA, Chu RH, Chandler DL, Beck RW, Holmes JM, Rice ML. Reliability of the electronic early treatment diabetic retinopathy study testing protocol in children 7 to $<13$ Years Old. Am J Ophthalmol 2003; 136: 655-661.

17 Moke PS, Turpin AH, Beck RW, Holmes JM, Repka MX, Birch EE. Computerized method of visual acuity testing: adaptation of the amblyopia treatment study visual acuity testing protocol. Am J Ophthalmol 2001; 132: 903-909.

18 Vanden Bosch ME, Wall M. Visual acuity scored by the letter-by-letter or probit methods has lower retest variability than the line assignment method. Eye 1997; 11: 411-417.

19 Bailey IL, Bullimore MA, Raasch TW, Taylor HR. Clinical grading and the effects of scaling. Invest Ophthalmol Vis Sci 1991; 32: 422-432.

20 Deming WE. Statistical Adjustment of Data. Wiley: New York, 1943.

21 Patton N, Aslam T, Murray G. Statistical strategies to assess reliability in ophthalmology. Eye 2006; 20(7): 749-754.

22 Dobson V, Clifford-Donaldson CE, Miller J, Garvey KA, Harvey EM. A comparison of Lea Symbols vs ETDRS letter distance visual acuity in a population of young children with a high prevalence of astigmatism. J AAPOS 2009; 13(3): 253-257.

23 Dobson V, Maguire M, Orel-Bixler D, Quinn G, Ying GS. Visual acuity results in school-aged children and adults: Lea Symbols chart vs Bailey-Lovie chart. Optom Vis Science 2003; 80(9): 650-654.

24 Carkeet A. Modeling logMAR visual acuity scores: effects of termination rules and alternative forced-choice options. Optom Vis Sci 2001; 78: 529-538.

25 Shah N, Dakin SC, Redmond T, Anderson RS. Vanishing Optotype acuity: Repeatability and effect of the number of alternatives. Ophthalmic Physiol Opt 2011; 31: 17-22. 